\title{
Microlensing by compact objects associated with gas clouds
}

\author{
V. Bozza ${ }^{1,5, \star}$, Ph. Jetzer ${ }^{2,3, \star \star}$, L. Mancini ${ }^{2,1,5, \star \star \star}$, and G. Scarpetta ${ }^{1,4,5, \dagger}$ \\ 1 Dipartimento di Fisica "E.R. Caianiello", Universitá di Salerno, 84081 Baronissi (SA), Italy \\ 2 Institut für Theoretische Physik der Universität Zürich, 8057 Zürich, Switzerland \\ 3 Institut für Theoretische Physik der ETH, 8093 Zürich, Switzerland \\ 4 International Institute for Advanced Scientific Studies, Vietri sul Mare (SA), Italy \\ ${ }^{5}$ Istituto Nazionale di Fisica Nucleare, sez. Napoli, Gruppo Collegato di Salerno, Italia
}

Received 4 May 2001 / Accepted 25 October 2001

\begin{abstract}
We investigate gravitational microlensing of point-like lenses surrounded by diffuse gas clouds. Besides gravitational bending, one must also consider refraction and absorption phenomena. According to the cloud density, the light curves may suffer small to large deviations from Paczyński curves, up to complete eclipses. Moreover, the presence of the cloud endows this type of microlensing event with a high chromaticity and absorption lines recognizable by spectral analysis. It is possible that these objects populate the halo of our galaxy, giving a conspicuous contribution to the fraction of the baryonic dark matter. The required features for the extension and the mass of the cloud to provide appreciable signatures are also met by several astrophysical objects.
\end{abstract}

Key words. gravitational lensing - ISM: clouds - stars: circumstellar matter - stars: pre-main sequence - ISM: planetary nebulae: general - stars: AGB and post-AGB

\section{Introduction}

In the last years gravitational microlensing has rapidly grown as an important astrophysical tool with wide applications. In particular, it provides some evidence for the presence of baryonic dark matter, in the form of compact objects (MACHOs), in the Galatic halo (Alcock et al. 2000a; Lasserre et al. 2000) although the number of microlensing events in the direction of the Magellanic Clouds, reported by the observational teams of MACHO and EROS, is lower by a factor $\sim 5$ of that predicted by the standard halo models. The estimated MACHO fraction is now about $20 \%$ of the total Galactic dark matter mass, though it must be kept in mind that this value is still affected by a very high uncertainty. On the other hand several hundred events have been found towards the Galactic bulge region (Alcock et al. 2000b; Woźniak et al. 2001) and few events towards some spiral arm regions (Derue et al. 2001). Most likely the lenses are in these cases low mass stars or brown dwarfs.

Classical microlensing consists of the magnification of the luminous flux coming from a star due to the presence of a deflecting mass close to the line of sight. Within

\footnotetext{
Send offprint requests to: V. Bozza,

* e-mail: valboz@sa.infn.it

$\star \star$ e-mail: jetzer@physik.unizh.ch

$\star \star \star$ e-mail: mancini@physik.unizh.ch

$\dagger$ e-mail: scarpetta@sa.infn.it
}

this picture, microlensing yields an indirect observational method for the detection of non-luminous bodies.

However, compact objects are not the only candidates for baryonic dark matter in the halo. Some years ago, it was proposed that an appreciable fraction of the Galactic dark matter might be in form of self-gravitating gas clouds with masses of the order of Jupiter's mass $\left(10^{-3} M_{\odot}\right)$ and radii $R \approx 10 \mathrm{AU}$ (Pfenninger et al. 1994; De Paolis et al. 1995a, 1995b, 1996, 1998a, 1998b; Gerhard \& Silk 1996). For diffuse objects two more phenomena must be taken into account. Traveling through a gaseous medium, light basically undergoes refraction and absorption. So, even if these gas clouds would produce no appreciable microlensing effect, classical refraction could supply an alternative amplification mechanism. The resulting light curves resemble gravitational microlensing ones, but can be distinguished by their peculiar features (Draine 1998; Rafikov \& Draine 2001).

In this paper, we mean to study an intermediate possibility, which might be of relevance for the estimate of baryonic dark matter in the halo. After almost ten years of microlensing campaigns, at a good confidence level, we can say that MACHOs exist. If gas clouds are effectively present in the halo, they could likely be associated with MACHOs. If this is the case, these Pointlike Lenses Associated with Gas (hereafter, PLAG) would be missed by standard microlensing observations, because their light curve would be significantly altered by the surrounding 
gas cloud. On the other hand, isolated gas clouds could be quite difficult to detect without a powerful amplification mechanism.

In PLAGs gravitational lensing by the central object would provide this mechanism, but the secondary effects due to the cloud would show up in a weak or strong fashion, depending on the density of the diffuse matter. Classical absorption by Rayleigh scattering would essentially block all light attempting to cross high density clouds. For lower densities, a moderate absorption would be contrasted by microlensing and refraction both contributing to the amplification of the flux coming from the source. In this case, a considerable reddening of the light must be expected, producing a high chromaticity in the light curve. Of course, the existence of the gas cloud could be revealed without doubt by taking a spectrum of the source during the microlensing event. In this way, the chemical composition of the matter surrounding the central compact object can be determined.

Several studies about the stability of gas clouds seem to favour the formation of a steady state in protostellar nebulae (Cazes \& Tohline 1998). If MACHOs were formed by the collapse of gas clouds, a large portion of this cold gas may still surround some of them, with a reasonable formation of PLAGs.

The abundance of dark PLAGs in the halo is not known, leaving open several possibilities. They could be completely absent, if some instability mechanism intervenes. They may be a negligible fraction of MACHOs, if their mean life is too small. On the contrary, they could be relatively abundant and partly explain the discrepancy between observations and theoretical predictions about the microlensing rate, since the missing microlensing events would be actually PLAG events, not detected by usual observations. If this is true, then microlensing experiences should be properly modified in order to detect PLAG events efficiently.

Besides dark PLAGs in the halo, many well-known astrophysical compact objects surrounded by gas are present in the galactic disk. We can mention, for instance, planetary and proto-planetary nebulae, proto-stars, LBV nebulae, nebular variables (T Tau stars and R W Aur stars), eruptive variables (R CrB stars, Wolf-Rayet stars, novae), and so on. Of course, not all of them are able to produce microlensing effects that are observable by monitoring programs, since their parameters should satisfy several constraints.

A fundamental tuning is required between the size of the cloud $R_{\mathrm{c}}$ and the Einstein radius $R_{\mathrm{E}}$. The ratio $R_{\mathrm{c}} / R_{\mathrm{E}}$ should be in the range $10^{-1} \div 10^{2}$. This tuning is demanded by the necessity of having a microlensing time comparable with the time required by the source to pass behind the whole gas cloud. In fact, if the cloud is too small, it would act, in all circumstances, as a compact object. On the contrary, if the cloud is much larger than the Einstein radius, with the typical velocities of galactic objects, the time taken for the source to pass behind the whole cloud may easily rise up to years. However, even in this case, these long duration events could be included among the objectives of observational campaigns following stars for very long periods.

For $R_{\mathrm{c}} / R_{\mathrm{E}} \gtrsim 10^{2}$, we would observe no deviation from the usual microlensing light curve, since the effects of the cloud would be manifest after decades and could not be noticed practically. No information on the size and the shape of the cloud would be retrieved in this case.

In Sect. 2, we introduce the basics of the three phenomena affecting the light passing through the cloud: gravitational lensing, refraction and absorption by Rayleigh scattering. In Sect. 3, we analyze the general features of the light curves expected from PLAGs. In Sect. 4, we briefly discuss the properties of the afore-mentioned PLAG candidates. Section 5 contains the summary.

\section{Light passing through gas clouds: Basic phenomena}

We restrict our analysis to spherical gas clouds surrounding point-like masses. This simplification allows an extensive analytical investigation. Moreover, spherically symmetric PLAGs should be easier to distinguish from variable stars and other usual backgrounds to microlensing.

The lens equation contains both refraction and gravitational lensing. The latter can be separated into two contributions: the deviation due to the central object and the one produced by the gas cloud. Thanks to the spherical symmetry, we can write the one dimensional equation

$y=x-\frac{D_{\mathrm{ol}} D_{\mathrm{ls}}}{D_{\mathrm{os}}}\left[\alpha_{0}(x)+\alpha_{\mathrm{c}}(x)+\alpha_{\mathrm{r}}(x)\right]$

where

$\alpha_{0}(x)=\frac{4 G M}{c^{2} x}$

is the deflection angle produced by the gravitational field of the compact object (of mass $M$ ) at the center of the PLAG (Schneider et al. 1992); $\alpha_{\mathrm{c}}(x)$ is the deviation angle due to the gravitational field of the gas cloud; $\alpha_{\mathrm{r}}(x)$ is the contribution to the deviation coming from refraction of light passing through the cloud. As usual, $y(x$, respectively) is the distance of the source (of the image, respectively) to the optical axis, defined as the line connecting the observer and the lens.

The splitting of the deviation angle into the sum of three separate contributions is possible in the linear approximation. Higher orders would mix up the three effects.

The gas cloud is described by a continuous density distribution $\rho(r)$ which is a function of the radial distance from the center of the PLAG, according to the spherical symmetry hypothesis. Since the PLAG has limited size, we call $R_{\mathrm{c}}$ the value of the radius where the density falls to zero. The total mass of the cloud is $M_{\mathrm{c}}$. The projected density is

$\Sigma(x)=\int_{-\sqrt{R_{\mathrm{c}}^{2}-x^{2}}}^{\sqrt{R_{\mathrm{c}}^{2}-x^{2}}} \rho\left(\sqrt{x^{2}+z^{2}}\right) \mathrm{d} z$. 
The gravitational bending for light rays passing outside the cloud $\left(x>R_{\mathrm{c}}\right)$ is just $\frac{4 G M_{\mathrm{c}}}{c^{2} x}$, while for $x<R_{\mathrm{c}}, \alpha_{\mathrm{c}}$ decreases to zero. It is generally given by

$\alpha_{\mathrm{c}}(x)=\frac{4 G}{c^{2}} \frac{2 \pi}{x} \int_{0}^{x} \Sigma\left(x^{\prime}\right) x^{\prime} \mathrm{d} x^{\prime}=\frac{4 G m(x)}{c^{2} x}$,

where $m(x)$ is the mass of the portion of the cloud contained in the cylinder of radius $x$ (Schneider et al. 1992).

Light rays passing inside the gas cloud undergo Rayleigh scattering by neutral gas (essentially hydrogenum and helium) and eventually dust. The macroscopic consequences are refraction and extinction, both depending on the density of the neutral gas $\rho_{\mathrm{n}}(r)$ and on its chemical composition.

The refraction index of a gas is

$n_{\lambda}(r)=1+\alpha_{\lambda} \rho_{\mathrm{n}}(r)$,

where $\alpha_{\lambda}$ is the specific refractivity. The deviation angle can be easily derived by the Fermat principle assuming the deviation to be small. Its expression is

$\alpha_{\mathrm{r}}(x)=-\alpha_{\lambda} \frac{\mathrm{d} \Sigma_{\mathrm{n}}}{\mathrm{d} x}=-2 \alpha_{\lambda} x \int_{x}^{R_{\mathrm{c}}} \frac{\mathrm{d} r}{\sqrt{r^{2}-x^{2}}} \frac{\mathrm{d} \rho_{\mathrm{n}}}{\mathrm{d} r}$.

Where $\Sigma_{\mathrm{n}}$ is the projected density of neutral gas (compare Draine 1998, Eq. (1)).

The magnification of the images follows from the one dimensional lens Eq. (1)

$\mu=\frac{x}{y \frac{\mathrm{d} y}{\mathrm{~d} x}}$

with $y$ given by the RHS of Eq. (1). This magnification factor is a consequence of the geometrical bending on the light rays, caused by refraction and gravitational field.

The observed intensity is moreover modified by combined effects of scattering and absorption, giving rise to the so-called extinction, described by Lambert's law

$I_{\lambda}=I_{\lambda, 0} \mathrm{e}^{-\tau_{\lambda}}$

where $I_{\lambda, 0}$ is the intensity that we see in absence of absorption along the line of sight, i.e. before and after the microlensing event, and $\tau_{\lambda}$ is the optical depth of the cloud, given by

$\tau_{\lambda}=k_{\lambda} \Sigma_{\mathrm{n}}(x)$

where $k_{\lambda}$ is the absorption coefficient.

Summing up, each image will have a total amplification given by the geometrical magnification (7) due to light bending (both gravitational and refractive) weighted by the transmission coefficient $\mathrm{e}^{-\tau_{\lambda}}$.

\section{General properties of PLAGs}

The fundamental parameters of the PLAG are the mass of the central object $M$, the radius of the cloud $R_{\mathrm{c}}$ and its mass $M_{\mathrm{c}}$. Most statements about the aspect of the light curves can be given in terms of these parameters, or their derived quantities, without need to specify the shape of the density profile (if it is smooth enough).

We define the Einstein radius

$R_{\mathrm{E}}=\sqrt{\frac{4 G\left(M+M_{\mathrm{c}}\right)}{c^{2}} \frac{D_{\mathrm{OL}} D_{\mathrm{LS}}}{D_{\mathrm{OS}}}}$

referring to the total mass of the system.

In this section, using a simplified model, we will discuss the phenomenology that should be generally expected in microlensing events caused by PLAGs.

\subsection{Sample family of PLAGs}

If we consider PLAGs formed by hot nebulae, in the central region the gas may be ionized, being uneffective for classical Rayleigh scattering, suggesting $\rho_{\mathrm{n}}<\rho$. We are mainly interested in cold dark PLAGs; so, in a first approach, we will assume that this region, if present, is small enough with respect to the total cloud and the Einstein radius, so that $\rho_{\mathrm{n}} \simeq \rho$. In this way, we can rapidly obtain a simple description of the basic phenomenology. Nevertheless, the extension to the case $\rho_{\mathrm{n}}<\rho$ is straightforward and can be easily faced successively.

The dependence on the nature of the gas is not so strong to substantially affect the discussion, since the order of magnitude of the specific refractivity and the absorption coefficient is the same for all the interesting elements or molecules. Thus, in all practical examples, we will assume a mixture of $\mathrm{H}_{2}$-He with $24 \%$ He by mass. At $\lambda=4400 \AA$ for this mixture, we have (AIP Handbook 1972; following Draine 1998)

$\alpha_{\lambda} \simeq 1.243 \mathrm{~cm}^{3} \mathrm{~g}^{-1}$

$k_{\lambda} \simeq 5.4 \times 10^{-5}\left(\frac{0.44 \mu \mathrm{m}}{\lambda}\right)^{4} \mathrm{~cm}^{2} \mathrm{~g}^{-1}$.

The variety of density profiles in nebulae is very large and most of them have no satisfying description at the present time. This variety seems to make the choice of the density model a hard task. However, once projected along the line of sight, all realistic density distributions are generally smoothly peaked at the center and decay towards the border in a more or less steep fashion. For this reason, the basic phenomenology of PLAG events does not depend on the specific shape of the original density profile but can be discussed by analyzing one representative model.

We choose the function

$\rho_{\beta}(r)=\left\{\begin{array}{ll}\rho_{\beta}^{0}\left(1-\frac{r^{2}}{R_{\mathrm{c}}^{2}}\right)^{\beta} & \text { for } r \leq R_{\mathrm{c}} \\ 0 & \text { otherwise }\end{array}\right.$,

parameterized by the exponent $\beta>0$, to describe the density profile of a typical PLAG. The choice of this model is strongly motivated by the fact that most physically interesting quantities can be calculated analytically starting from this distribution, while all the general features of a PLAG microlensing event can be obtained. Moreover, the parameter $\beta$ can be adjusted to suit distinct physical 
situations: high $\beta$ s would produce clouds concentrated at the center with smooth boundaries which would appropriately describe stable or nearly stable systems; low $\beta \mathrm{s}$, on the other hand, can be used for expanding clouds, which are generally characterized by a steep boundary and where abrupt changes in the density may produce discontinuities in the refraction angle (see Sect. 3.4 and Fig. 1b).

Of course, if one is interested in the details of the microlensing light curves, it is necessary to know the real shape of the nebula; but for all the highlights of a microlensing light curve, the study of this sample model will be sufficient.

The central density can be expressed in terms of the mass of the cloud

$M_{\mathrm{c}}=\int_{0}^{R_{\mathrm{c}}} 4 \pi r^{2} \rho_{\beta}(r) \mathrm{d} r=\rho_{\beta}^{0} \frac{\pi^{3 / 2} R_{\mathrm{c}}^{3} \Gamma[\beta+1]}{\Gamma\left[\beta+\frac{5}{2}\right]}$.

The projected density is

$\Sigma_{\beta}(x)=\rho_{\beta}^{0} \frac{\pi^{1 / 2} R_{\mathrm{c}} \Gamma[\beta+1]}{\Gamma\left[\beta+\frac{3}{2}\right]}\left(1-\frac{x^{2}}{R_{\mathrm{c}}^{2}}\right)^{\beta+\frac{1}{2}}$.

The gravitational bending due to this density distribution is

$\alpha_{\mathrm{c}}(x)=\frac{4 G \rho_{\beta}^{0} \pi^{3 / 2} R_{\mathrm{c}}^{3} \Gamma[\beta+1]}{c^{2} x \Gamma\left[\beta+\frac{5}{2}\right]}\left[1-\left(1-\frac{x^{2}}{R_{\mathrm{c}}^{2}}\right)^{\beta+\frac{3}{2}}\right]$.

The refraction angle is

$\alpha_{\mathrm{r}}(x)=\alpha_{\lambda} \frac{2 \beta \rho_{\beta}^{0} \pi^{1 / 2} R_{\mathrm{c}}^{3} \Gamma[\beta]}{\Gamma\left[\beta+\frac{1}{2}\right]} \frac{x}{R_{\mathrm{c}}}\left(1-\frac{x^{2}}{R_{\mathrm{c}}^{2}}\right)^{\beta-\frac{1}{2}}$.

Of course, we cannot take as physical the divergence for $0<\beta<1 / 2$. The simple expression we are using for the refraction angle is valid for small deviations, so the presence of such divergence signals a breakdown of this hypothesis and the need for higher order corrections. These corrections should provide a cutoff for the refraction angle.

In Fig. 1 we plot the gravitational bending, the refraction angle and the transmission coefficient of our cloud model for $M_{\mathrm{c}}=0.01 M_{\odot}, R_{\mathrm{c}}=6 \mathrm{AU}$ and for different values of $\beta$.

\subsection{Light curves}

We can distinguish two main classes of PLAGs, according to the ratio between $R_{\mathrm{c}}$ and $R_{\mathrm{E}}$.

In small PLAGs $\left(R_{\mathrm{c}}<R_{\mathrm{E}}\right)$, the positive parity image (the image formed outside of the Einstein ring) is not affected by the existence of the gas cloud. Its position is just that of the principal image of a point-like lens with mass $M+M_{\mathrm{c}}$. Since light passes outside of the cloud, neither refraction nor absorption modify the simple gravitational lensing effect.

The secondary image has negative parity and is formed inside the Einstein ring. If the source is close enough to the optical axis, this image is external to the cloud as well. In this situation, the PLAG acts as a point-like lens at all
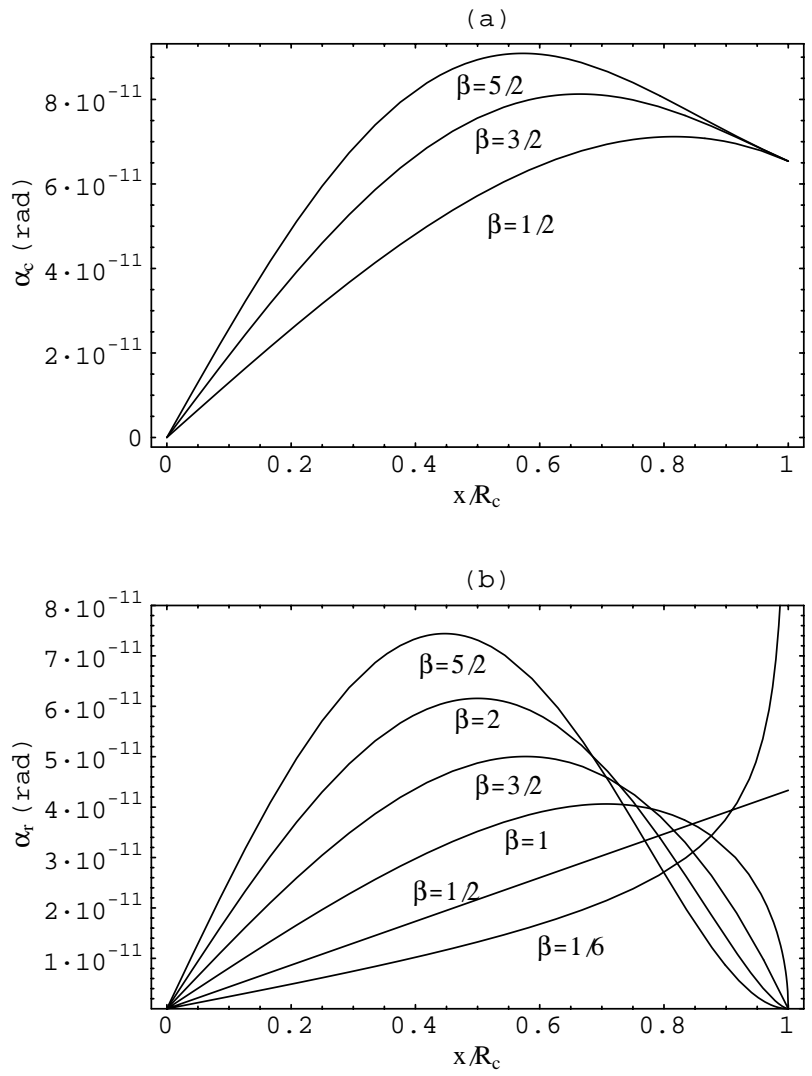

(c)

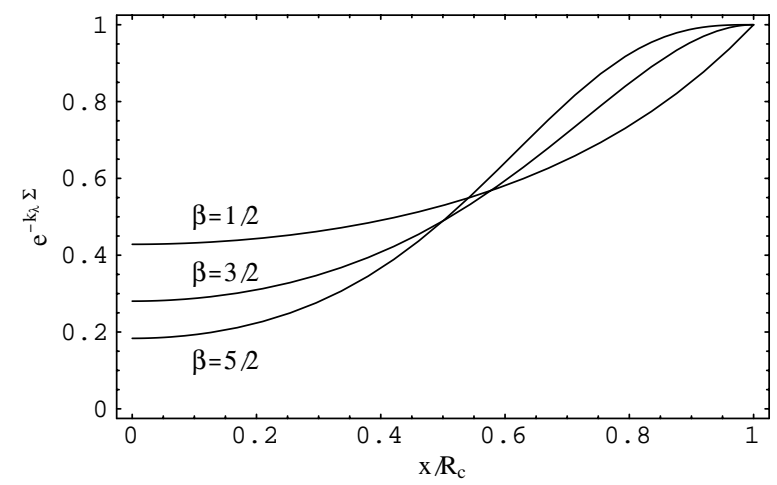

Fig. 1. a) Gravitational bending due to the cloud. b) Refraction angle. c) Transmission coefficient. All quantities are plotted for different values of $\beta$.

effects. When the source is far from the optical axis, the secondary image is formed inside the PLAG. In this case, its position is modified by the non-trivial form of the deviation angles $\alpha_{\mathrm{r}}$ and $\alpha_{\mathrm{c}}$. Moreover, the light flux coming from this image is partially absorbed. However, the secondary image is relevant for the total flux only when it is close to the Einstein ring, otherwise it is highly demagnified. So, these additional effects intervene in a regime where the image does not contribute much to the light curve. On the basis of these considerations, the typical light curve we expect for $R_{\mathrm{c}}<R_{\mathrm{E}}$ is a Paczyński-like curve slightly lowered far from the maximum, where the secondary image enters the cloud. This deviation can be easily confused in the background photometric noise. 
(a)

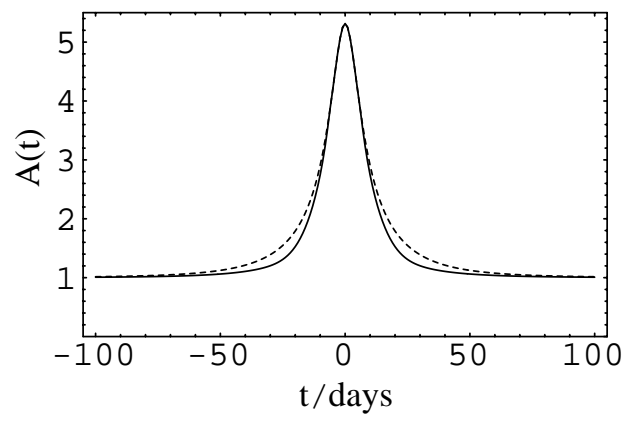

(c)

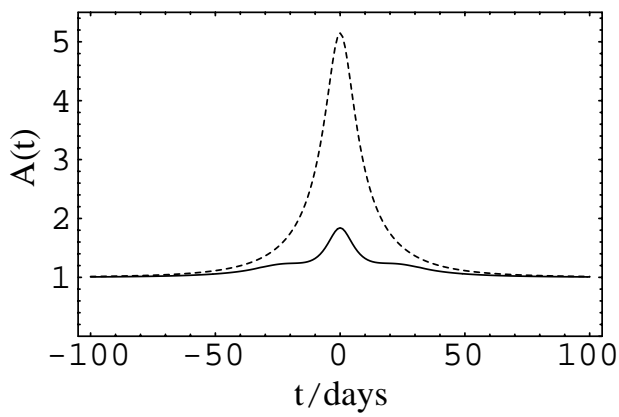

(e)

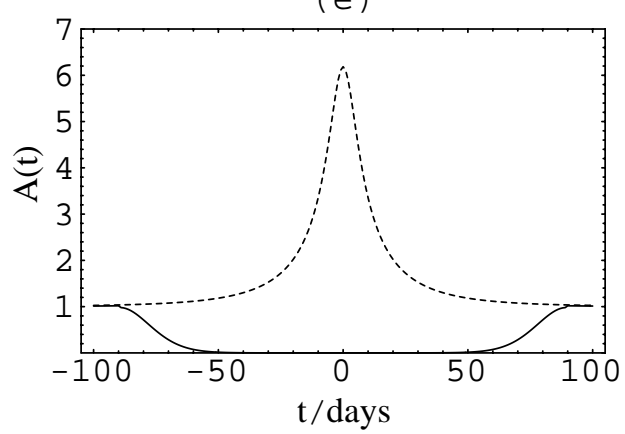

(b)

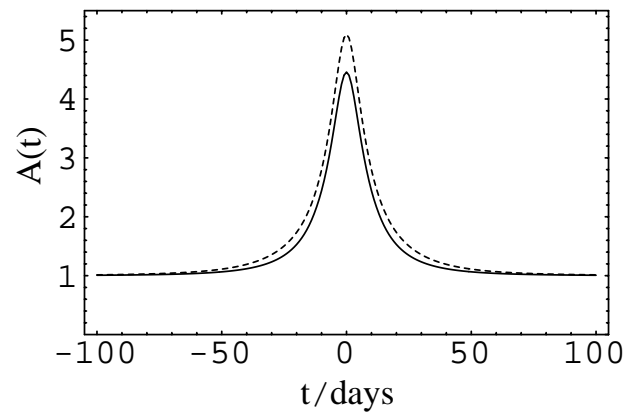

(d)

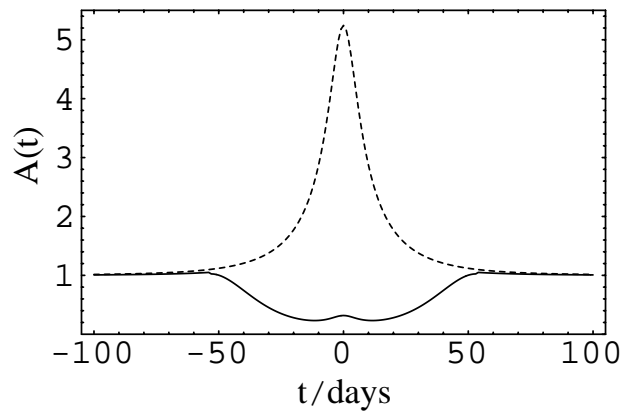

(f)

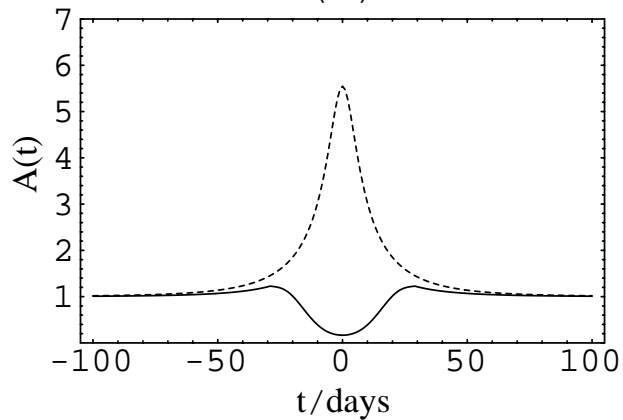

Fig. 2. The six categories of light curves expected for PLAG events as discussed in Sect. 3.2. The dotted lines represent the classical Paczyński light curves for gravitational lensing by the total mass $M+M_{\mathrm{c}}$. The common parameters, typical for a disk/bulge microlensing events, are $\beta=5 / 2, D_{\mathrm{ol}}=8 \mathrm{kpc}, D_{\mathrm{ls}}=2 \mathrm{kpc}, M=1 M_{\odot}$, impact parameter $b=0.2 R_{\mathrm{E}}$ and transverse velocity $v_{\mathrm{T}}=200 \mathrm{~km} \mathrm{~s}^{-1}$. The other parameters are: a) $\left.\left.M_{\mathrm{c}}=0.01 M_{\odot}, R_{\mathrm{c}}=3.3 \mathrm{AU} ; \mathbf{b}\right) M_{\mathrm{c}}=0.01 M_{\odot}, R_{\mathrm{c}}=4.5 \mathrm{AU} ; \mathbf{c}\right)$ $\left.\left.M_{\mathrm{c}}=0.03 M_{\odot}, R_{\mathrm{c}}=6 \mathrm{AU} ; \mathbf{d}\right) M_{\mathrm{c}}=0.7 M_{\odot}, R_{\mathrm{c}}=8 \mathrm{AU} ; \mathbf{e}\right) M_{\mathrm{c}}=0.5 M_{\odot}, R_{\mathrm{c}}=12 \mathrm{AU} ;(\mathrm{f}) M_{\mathrm{c}}=0.2 M_{\odot}, R_{\mathrm{c}}=6 \mathrm{AU}$.

To ensure the effect to be appreciable, the size of the cloud should be as close as possible to the Einstein ring and the density as large as possible. In fact, in this case, the secondary image would remain inside the cloud during a large fraction of the microlensing event. A large absorption would depress it until it exits from the cloud. So, just the region in the neighborhood of the peak would be purely microlensing, while outside of this interval, the secondary image would be depressed, changing the shape of the curve. An example is given in Fig. 2a, drawn (like the others) using our sample model.

The time of the entrance of the secondary image into the cloud is

$t=t_{0}+\frac{1}{v_{\mathrm{T}}} \sqrt{\left(\frac{R_{\mathrm{E}}^{2}}{R_{\mathrm{c}}}-R_{\mathrm{c}}\right)-b^{2}}$

where $b$ is the impact parameter of the source to the optical axis, $v_{\mathrm{T}}$ is the relative projected velocity and $t_{0}$ is the time of the closest approach.

An interesting phenomenology arises when $R_{\mathrm{c}}>R_{\mathrm{E}}$. The secondary image is always inside the cloud, being displaced and absorbed. The principal image would enter the cloud only in the neighborhood of $t_{0}$, for sufficiently small impact parameters. The time of the exit of the principal image from the cloud is still given by Eq. (18). Depending on the density of the cloud, we can distinguish some cases.

If the density is low, the absorption slightly lowers the maximum with respect to Paczyński (Fig. 2b).

For moderate densities, the curve will be depressed when the principal image enters the cloud. So, during the ascent to the maximum, the curve will suddenly decrease its derivative and then complete its rise to a considerably smaller maximum (Fig. 2c).

For high densities, the absorption will prevail over the magnification. When the principal image enters the cloud, 
Table 1. Maximal fractional contribution to the complete light curve coming from the gravitational bending due to the cloud.

\begin{tabular}{l|ccccccc}
\hline \hline & $\frac{R_{\mathrm{c}}}{R_{\mathrm{E}}}=0.5$ & $\frac{R_{\mathrm{c}}}{R_{\mathrm{E}}}=0.75$ & $\frac{R_{\mathrm{c}}}{R_{\mathrm{E}}}=1$ & $\frac{R_{\mathrm{c}}}{R_{\mathrm{E}}}=1.25$ & $\frac{R_{\mathrm{c}}}{R_{\mathrm{E}}}=1.5$ & $\frac{R_{\mathrm{c}}}{R_{\mathrm{E}}}=1.75$ & $\frac{R_{\mathrm{C}}}{R_{\mathrm{E}}}=2$ \\
\hline$M_{\mathrm{c}} / M=10^{-4}$ & $4.9 \times 10^{-5}$ & $4.9 \times 10^{-5}$ & $5 \times 10^{-5}$ & $6 \times 10^{-5}$ & $7.2 \times 10^{-5}$ & $7.6 \times 10^{-5}$ & $7.4 \times 10^{-5}$ \\
$M_{\mathrm{c}} / M=10^{-3.5}$ & $1.5 \times 10^{-4}$ & $1.5 \times 10^{-4}$ & $1.6 \times 10^{-4}$ & $1.9 \times 10^{-4}$ & $2.3 \times 10^{-4}$ & $2.4 \times 10^{-4}$ & $2.4 \times 10^{-4}$ \\
$M_{\mathrm{c}} / M=10^{-3}$ & $4.8 \times 10^{-4}$ & $4.8 \times 10^{-4}$ & $5.1 \times 10^{-4}$ & $6.5 \times 10^{-4}$ & $7.7 \times 10^{-4}$ & $8 \times 10^{-4}$ & $7.6 \times 10^{-4}$ \\
$M_{\mathrm{c}} / M=10^{-2.5}$ & $1.5 \times 10^{-3}$ & $1.5 \times 10^{-3}$ & $1.8 \times 10^{-3}$ & $2.4 \times 10^{-3}$ & $2.9 \times 10^{-3}$ & $2.9 \times 10^{-3}$ & $2.6 \times 10^{-3}$ \\
$M_{\mathrm{c}} / M=10^{-2}$ & $4.8 \times 10^{-3}$ & $5 \times 10^{-3}$ & $7.4 \times 10^{-3}$ & $1.1 \times 10^{-2}$ & $1.3 \times 10^{-2}$ & $1.2 \times 10^{-2}$ & $10^{-2}$ \\
$M_{\mathrm{c}} / M=10^{-1.5}$ & $1.5 \times 10^{-2}$ & $2.2 \times 10^{-2}$ & $3.3 \times 10^{-2}$ & $5.8 \times 10^{-2}$ & $7.4 \times 10^{-2}$ & $6.6 \times 10^{-2}$ & $5.2 \times 10^{-2}$ \\
$M_{\mathrm{c}} / M=10^{-1}$ & $4.5 \times 10^{-2}$ & $9.6 \times 10^{-2}$ & 0.13 & 0.28 & 0.4 & 0.37 & 0.29 \\
$M_{\mathrm{c}} / M=10^{-0.5}$ & 0.12 & 0.26 & 0.42 & 0.83 & 0.95 & 0.95 & 0.90 \\
\hline
\end{tabular}

Table 2. Maximal fractional contribution to the complete light curve coming from refraction.

\begin{tabular}{l|ccccccc}
\hline \hline & $\frac{R_{\mathrm{c}}}{R_{\mathrm{E}}}=0.5$ & $\frac{R_{\mathrm{c}}}{R_{\mathrm{E}}}=0.75$ & $\frac{R_{\mathrm{c}}}{R_{\mathrm{E}}}=1$ & $\frac{R_{\mathrm{c}}}{R_{\mathrm{E}}}=1.25$ & $\frac{R_{\mathrm{c}}}{R_{\mathrm{E}}}=1.5$ & $\frac{R_{\mathrm{c}}}{R_{\mathrm{E}}}=1.75$ & $\frac{R_{\mathrm{c}}}{R_{\mathrm{E}}}=2$ \\
\hline$M_{\mathrm{c}} / M=10^{-4}$ & $5.7 \times 10^{-5}$ & $9.3 \times 10^{-5}$ & $9.9 \times 10^{-5}$ & $10^{-4}$ & $3.8 \times 10^{-5}$ & $2.9 \times 10^{-5}$ & $2.9 \times 10^{-5}$ \\
$M_{\mathrm{c}} / M=10^{-3.5}$ & $1.8 \times 10^{-4}$ & $2.9 \times 10^{-4}$ & $3.1 \times 10^{-4}$ & $3.3 \times 10^{-4}$ & $1.2 \times 10^{-4}$ & $9.3 \times 10^{-5}$ & $9.3 \times 10^{-5}$ \\
$M_{\mathrm{c}} / M=10^{-3}$ & $5.5 \times 10^{-4}$ & $9.1 \times 10^{-4}$ & $9.8 \times 10^{-4}$ & $10^{-3}$ & $3.8 \times 10^{-4}$ & $3 \times 10^{-4}$ & $3.1 \times 10^{-4}$ \\
$M_{\mathrm{c}} / M=10^{-2.5}$ & $1.6 \times 10^{-3}$ & $2.8 \times 10^{-3}$ & $3 \times 10^{-3}$ & $3 \times 10^{-3}$ & $1.2 \times 10^{-3}$ & $10^{-3}$ & $1.1 \times 10^{-3}$ \\
$M_{\mathrm{c}} / M=10^{-2}$ & $4.3 \times 10^{-3}$ & $7.9 \times 10^{-3}$ & $8.9 \times 10^{-3}$ & $7.3 \times 10^{-3}$ & $4.3 \times 10^{-3}$ & $5 \times 10^{-3}$ & $4.5 \times 10^{-3}$ \\
$M_{\mathrm{c}} / M=10^{-1.5}$ & $9.5 \times 10^{-3}$ & $2 \times 10^{-2}$ & $2.3 \times 10^{-2}$ & $1.3 \times 10^{-2}$ & $2.8 \times 10^{-2}$ & $3.3 \times 10^{-2}$ & $2.5 \times 10^{-2}$ \\
$M_{\mathrm{c}} / M=10^{-1}$ & $1.6 \times 10^{-2}$ & $4.3 \times 10^{-2}$ & $4 \times 10^{-2}$ & $6.4 \times 10^{-2}$ & 0.2 & 0.22 & 0.16 \\
$M_{\mathrm{c}} / M=10^{-0.5}$ & $2.5 \times 10^{-2}$ & $7.1 \times 10^{-2}$ & $7.4 \times 10^{-2}$ & 0.18 & 0.76 & 0.82 & 0.7 \\
\hline
\end{tabular}

Table 3. Maximal fractional absorption.

\begin{tabular}{l|ccccccc}
\hline \hline & $\frac{R_{\mathrm{c}}}{R_{\mathrm{E}}}=0.5$ & $\frac{R_{\mathrm{c}}}{R_{\mathrm{E}}}=0.75$ & $\frac{R_{\mathrm{c}}}{R_{\mathrm{E}}}=1$ & $\frac{R_{\mathrm{c}}}{R_{\mathrm{E}}}=1.25$ & $\frac{R_{\mathrm{c}}}{R_{\mathrm{E}}}=1.5$ & $\frac{R_{\mathrm{c}}}{R_{\mathrm{E}}}=1.75$ & $\frac{R_{\mathrm{c}}}{R_{\mathrm{E}}}=2$ \\
\hline$M_{\mathrm{c}} / M=10^{-4}$ & $3.8 \times 10^{-4}$ & $8.2 \times 10^{-4}$ & $1.3 \times 10^{-3}$ & $1.8 \times 10^{-3}$ & $3.2 \times 10^{-3}$ & $4.2 \times 10^{-3}$ & $4.5 \times 10^{-3}$ \\
$M_{\mathrm{c}} / M=10^{-3.5}$ & $1.1 \times 10^{-3}$ & $2.6 \times 10^{-3}$ & $4.2 \times 10^{-3}$ & $5.7 \times 10^{-3}$ & $10^{-2}$ & $1.3 \times 10^{-2}$ & $1.4 \times 10^{-2}$ \\
$M_{\mathrm{c}} / M=10^{-3}$ & $3.2 \times 10^{-3}$ & $7.6 \times 10^{-3}$ & $1.3 \times 10^{-2}$ & $1.7 \times 10^{-2}$ & $3.1 \times 10^{-2}$ & $4.1 \times 10^{-2}$ & $4.4 \times 10^{-2}$ \\
$M_{\mathrm{c}} / M=10^{-2.5}$ & $7.4 \times 10^{-3}$ & $2 \times 10^{-2}$ & $3.6 \times 10^{-2}$ & $5.1 \times 10^{-2}$ & $9.5 \times 10^{-2}$ & 0.12 & 0.13 \\
$M_{\mathrm{c}} / M=10^{-2}$ & $1.2 \times 10^{-2}$ & $4.3 \times 10^{-2}$ & $8.6 \times 10^{-2}$ & 0.13 & 0.26 & 0.34 & 0.36 \\
$M_{\mathrm{c}} / M=10^{-1.5}$ & $1.3 \times 10^{-2}$ & $6.4 \times 10^{-2}$ & 0.15 & 0.29 & 0.57 & 0.7 & 0.74 \\
$M_{\mathrm{c}} / M=10^{-1}$ & $9.9 \times 10^{-3}$ & $6.5 \times 10^{-2}$ & 0.19 & 0.49 & 0.86 & 0.96 & 0.98 \\
$M_{\mathrm{c}} / M=10^{-0.5}$ & $6.1 \times 10^{-3}$ & $5 \times 10^{-2}$ & 0.18 & 0.62 & 0.98 & 0.9991 & 0.9999 \\
\hline
\end{tabular}

the light reaching the observer falls rapidly. Eventually, the magnification at $t=t_{0}$ can still produce a low central peak in the valley (Fig. 2d), but for higher densities, even this faint feature would disappear. If the time of the entrance is far from $t_{0}$, the event resolves in a complete eclipse of the source (Fig. 2e); otherwise, the initial part of the ascent can signal the presence of the central lens inside the cloud (Fig. 2f).

The curves just described represent what can be generally expected from this physical situation. As already quoted, the specific shape obviously depends on the density profile considered. This fact can be exploited to try to achieve a reconstruction of the density profile from the details of the microlensing light curve.
We can make some simple considerations about the relative weight of gravitational lensing, refraction and absorption in the light curves, $A(t)$. Keeping $\beta=5 / 2$, we have compared the partial light curves (obtained neglecting one of the above-quoted phenomena at a time) with the exact ones. First, for each value of $M_{\mathrm{c}} / M$ and $R_{\mathrm{c}} / R_{\mathrm{E}}$, we have calculated the fractional deviation of the curve without cloud gravitational lensing with respect to the complete one, in the course of the whole microlensing event

$f_{\alpha_{\mathrm{c}}=0}(t)=\frac{|A(t)-A(t)|_{\alpha_{\mathrm{c}}=0} \mid}{A(t)}$.

In Table 1 we report the maximal deviation defined as $\max \left\{f_{\alpha_{\mathrm{c}}=0}(t)\right\}$ occurring in each curve. In the same way, 
in Table 2, we have collected the maximal fractional deviation of the curves without cloud refraction $\alpha_{\mathrm{r}}$ with respect to the complete ones. Table 3 contains the maximal fractional absorption. All light curves were computed assuming an impact parameter $b=0.2 R_{\mathrm{E}}, M=1 M_{\odot}$ and with $\lambda=4400 \AA$.

With these tables it is easy to distinguish the regimes where the different phenomena become important, keeping the gravitational lensing of the central object as a reference.

Cloud gravitational lensing can be neglected when the cloud mass is below a few hundredth of the central mass. Its effects become heavier for higher cloud masses, maximally for radii of the order $R_{\mathrm{c}} / R_{\mathrm{E}} \sim 1.5$.

Refraction is always of the same order as cloud gravitational lensing, dominating only for small radii. It is not a surprise that the sum of the fractional deviations due to refraction and gravitational lensing is sometimes greater than one. In fact, their action is by no way additive, since they simultaneously concur in the shift of the images and modify the magnification formula in a non-linear way.

All ranges of the cloud parameters where the refraction contribution is non-negligible are also affected by a large absorption. High masses completely block the light as we can read in the last row of Table 3. Cloud masses around one tenth of the central mass still show good positive deviations coming from light bending, maintaining a moderate absorption. It is in this mass range that PLAGs have the highest probability of being detected.

\subsection{Chromaticity}

Both refraction and absorption have a moderate dependence on the wavelength of the incoming wave. This means that a strong chromaticity should be expected for PLAG events, mostly caused by a selective absorption. In a minor extent, blending and refraction may slightly modify the color. Given the $\lambda^{-4}$ dependence of the absorption coefficient, Eq. (12), the light filtered through the PLAG would be strongly reddened, as it is evident from Fig. 3, where we reported the light curve of the example treated in Fig. 2c at different wavelenghts: $\lambda=4400 \AA$ (blue), Fig. 3a, and $\lambda=7000 \AA$ (red), Fig. 3b. For this reason, the achromaticity test of classical microlensing surveys would automatically reject PLAG events and should be relaxed in order to find them. Moreover, the detection of a PLAG would be helped by choosing filters operating in opposite bands of the spectrum so as to show the real nature of the chromaticity in an optimal way.

\subsection{Boundary discontinuities}

In expanding shells produced by old stars, the density profile is generally characterized by a steep boundary, where the density suddenly changes from zero to a value significant for refraction and absorption effects. Of course, this is not the case for cold dark PLAGs on which we are

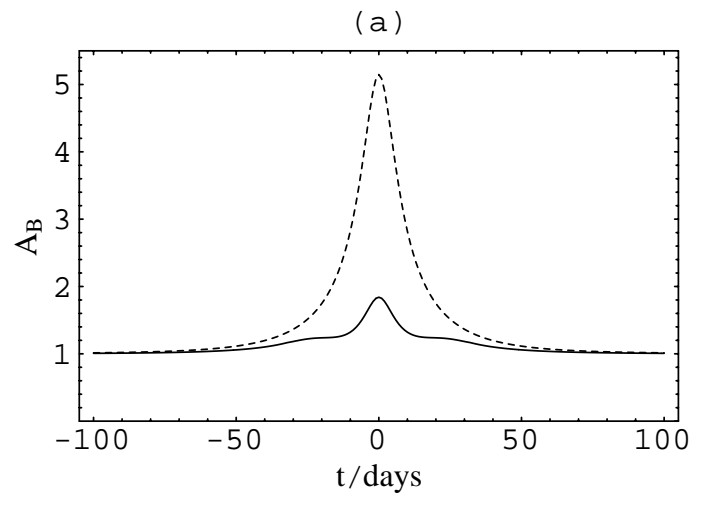

(b)

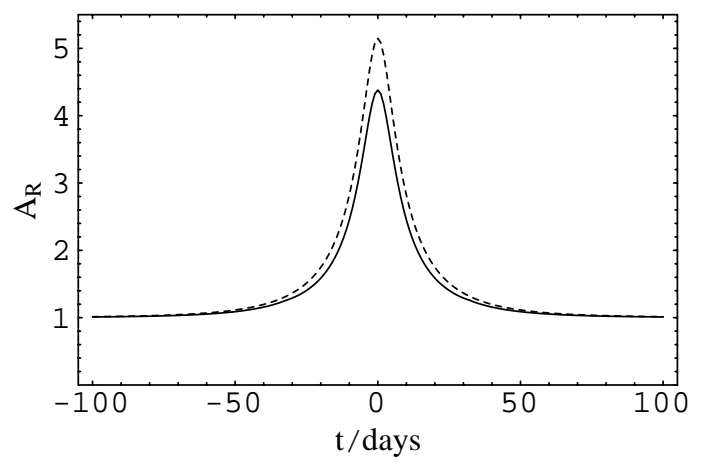

(c)

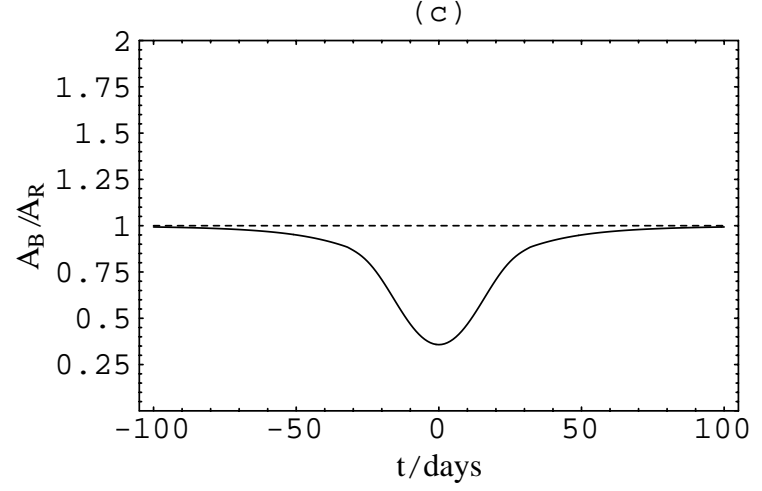

Fig. 3. Chromaticity of the PLAG microlensing event. a) $\lambda=$ $4400 \AA$ (blue). b) $\lambda=7000 \AA$ (red). The dotted lines are again the classical Paczyński light curves. c) Ratio between the two curves.

mainly focused in this article, which are supposed to be stable or long-lived systems; a wide range of alternative PLAG candidates (see Sect. 4) require an appropriate description of boundary effects.

For this reason, in this subsection we check whether interesting observable effects could be expected when one of the images enters an expanding steep-front cloud.

First of all, let us try a perturbative resolution of the lens equation for $x \simeq R_{\mathrm{c}}$. We let

$y=R_{\mathrm{c}}-\frac{R_{\mathrm{E}}^{2}}{R_{\mathrm{c}}}-\delta y$

and

$x=R_{\mathrm{c}}-\varepsilon$. 
In the neighborhood of $R_{\mathrm{c}}, \rho_{\beta}(r)$ falls to zero as

$\rho_{\beta}(r)=f(r)\left(1-\frac{r}{R_{\mathrm{c}}}\right)^{\beta}$

with $f(r)$ a regular non vanishing function at $r=R_{\mathrm{c}}$. This expansion can be performed in general, not only for the sample family studied so far, but also for a generic density profile.

The projected density will go as

$\Sigma_{\beta} \simeq 2 \sqrt{2} f\left(R_{\mathrm{c}}\right) \frac{\varepsilon^{\beta+\frac{1}{2}}}{R_{\mathrm{c}}^{\beta-\frac{1}{2}}}$.

Expanding all deviation angles to a first order in $\varepsilon$, we get

$\alpha_{0} \simeq \frac{R_{\mathrm{E}}^{2}}{R_{\mathrm{c}}} \frac{M}{M+M_{\mathrm{c}}}\left(1+\frac{\varepsilon}{R_{\mathrm{c}}}\right)$

$\alpha_{c} \simeq \frac{R_{\mathrm{E}}^{2}}{R_{\mathrm{c}}} \frac{M_{\mathrm{c}}}{M+M_{\mathrm{c}}}\left(1+\frac{\varepsilon}{R_{\mathrm{c}}}\right)$

$\alpha_{\mathrm{r}} \simeq \alpha_{\lambda} 2 \sqrt{2} f\left(R_{\mathrm{c}}\right)\left(\beta+\frac{1}{2}\right) \frac{\varepsilon^{\beta-\frac{1}{2}}}{R_{\mathrm{c}}^{\beta-\frac{1}{2}}}=A \varepsilon^{\beta-\frac{1}{2}}$,

which can be taken seriously only for $\beta \geq \frac{1}{2}$. For lower values, a cut-off from higher order terms should be expected.

The perturbed lens equation reads

$\delta y=\varepsilon+A \varepsilon^{\beta-\frac{1}{2}}+\frac{R_{\mathrm{E}}^{2}}{R_{\mathrm{c}}^{2}} \varepsilon$

and the magnification for an image close to the boundary is

$$
\begin{aligned}
\mu= & {\left[1-\frac{R_{\mathrm{E}}^{2}}{R_{\mathrm{c}}^{2}}\left(1+\frac{2 \varepsilon}{R_{\mathrm{c}}}\right)-\frac{A}{R_{\mathrm{c}}} \varepsilon^{\beta-\frac{1}{2}}\right]^{-1} } \\
& \times\left[1+\frac{R_{\mathrm{E}}^{2}}{R_{\mathrm{c}}^{2}}\left(1+\frac{2 \varepsilon}{R_{\mathrm{c}}}\right)+\left(\beta-\frac{1}{2}\right) A \varepsilon^{\beta-\frac{3}{2}}\right]^{-1} .
\end{aligned}
$$

Refraction yields $\beta$-dependent terms in the lens equation and the magnification. It is easy to analyze the boundary effects that may arise for low values of $\beta$. In Fig. 4 , the light curve is shown for different values of $\beta$ at the time of the entrance of an image inside the cloud.

According to the value of $\beta$, we can have different cases.

1. $\beta>5 / 2$ : the refraction terms introduce subdominant corrections. The magnification is a continuous function.

2. $\beta=5 / 2$ : the first derivative of the magnification has a discontinuity, being higher for $r<R_{\mathrm{c}}$. The light curve suddenly decreases its derivative when an image enters the cloud.

3. $3 / 2<\beta<5 / 2$ : the refraction term becomes dominant in the magnification. The derivative of the magnification goes to infinity in $R_{\mathrm{c}}$ and the light curve has a continuous depression when an image comes into the cloud.

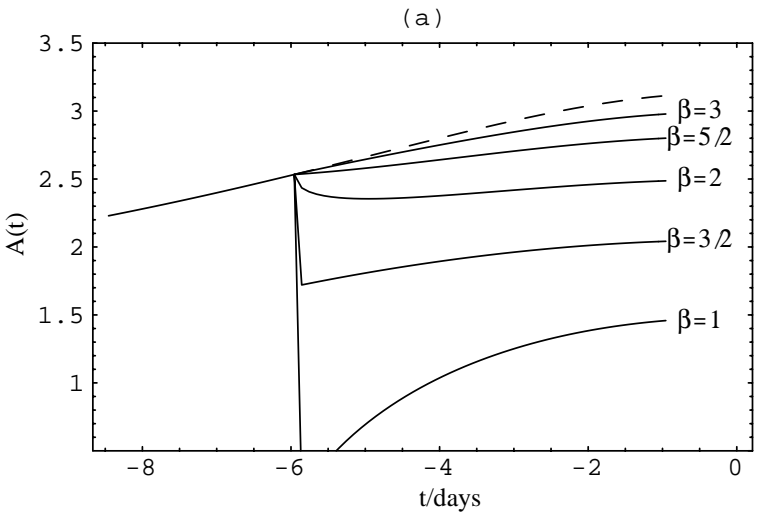

(b)

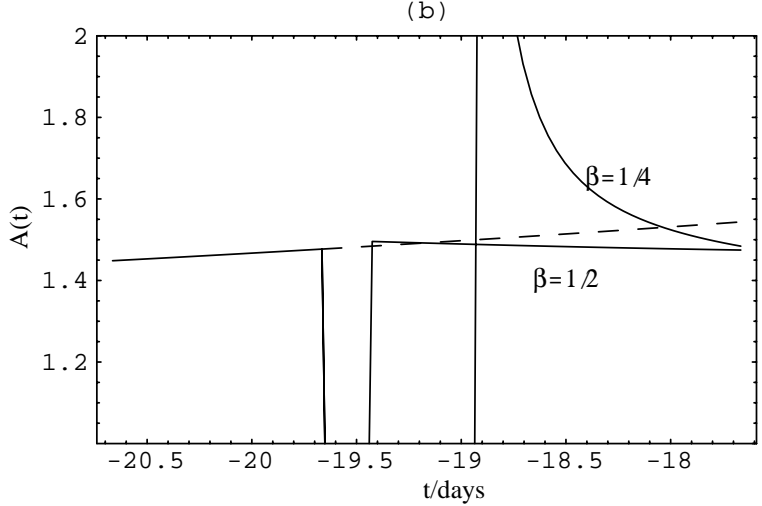

Fig. 4. Details of light curves at the entrance of an image into the cloud. The dashed curve is the classical Paczyński. a) Plots for $\beta=3,5 / 2,2,3 / 2,1$. b) Plots for $\beta=1 / 2,1 / 4$.

4. $\beta=3 / 2$ : the refraction terms in the magnification do not vanish on the border. The light curve has a finite discontinuity.

5. $1 / 2<\beta<3 / 2$ : the refraction term in the second square brackets of Eq. (28) diverges and the magnification falls off to zero. Then it rises from zero to the value it would have without refraction.

6. $\beta=1 / 2$ : the lens equation has no solution until $\delta y$ becomes greater than $A$. This fact provokes a hole in the light curve at the entrance of the image into the cloud, illustrated in Fig. 4b for our sample model with $\beta=1 / 2$.

7. $0 \leq \beta<1 / 2$ : again we have a hole in the light curve. Increasing $\delta y$ further, two values of $\varepsilon$ solve the equation. One approaches the original linear relation, behaving as the continuation of the original image. The other approaches zero, i.e. an additional image is formed very close to the border of the cloud.

So, when an image enters the cloud, after a short interval where it temporarily disappears, it comes out doubled, with the additional image staying close to the border. The formation of this pair happens on a radial critical curve ${ }^{1}$ and the magnification diverges at the formation of the two images.

${ }^{1}$ We recall that the critical curves are defined by the vanishing of the Jacobian determinant of the lens application. In axisymmetric lenses a critical curve is tangential if $y(x)=0$, while is radial if $\frac{\partial y}{\partial x}=0$. 
These boundary features produced by refraction are a very interesting tool to investigate the shape and the nature of the cloud; but they last only a few hours and would be hard to detect. It must be recalled that for such steep variations the finite size of the source intervenes to smooth peaks and holes. Looking at the their typical time scales, these features would not be more elusive than planetary microlensing, after all. For very sharp density profiles, higher order corrections in the refraction formula should be taken into account for a correct description at very low $\beta$.

\subsection{Additional critical curves}

In the previous subsection, we have seen that the structure of the Jacobian can be predicted once we know the expansion of the density distribution near the boundary (22). In this way, we have found that refraction would produce radial critical curves very close to the boundary in densities with very steep fronts ${ }^{2}$. Are these the only possible additional critical curves?

In isolated clouds, without the central object, critical curves and caustics can be produced when the density is sufficiently high, as discussed by Draine (1998). However, when a central mass is present and the cloud mass is nondominant with respect to the central mass, it is far more difficult to create critical curves, as we shall discuss in the following.

To have a tangential critical curve, we need the RHS of the lens Eq. (1) to vanish. Usually the Einstein ring is the only critical curve of this class. It is difficult to have additional curves inside the Einstein ring, because we would need a very highly negative refraction angle to compensate the other terms. This kind of refraction angle could only come out in shell densities with a very steep internal front. Additional tangential critical curves can be created outside of the Einstein ring with very high positive refraction angles. Such angles are beyond the reach of a linear theory and higher order terms would cut off the refraction angle excluding the formation of tangential critical curves.

The situation is similar for radial critical curves. Here we have to kill the derivative of the RHS of (1), i.e. we have to satisfy the equation

$$
\begin{aligned}
1+ & \frac{D_{\mathrm{ol}} D_{\mathrm{ls}}}{D_{\mathrm{os}}}\left[\alpha_{\lambda} \Sigma^{\prime \prime}(x)\right. \\
& \left.+\frac{4 G}{c^{2}}\left(\frac{M+m(x)}{x^{2}}-2 \pi \Sigma(x)\right)\right]=0 .
\end{aligned}
$$

Without the refraction term, this condition reduces to the usual gravitational lensing one (Subramanian \& Cowling 1986). Refraction helps to reach this condition but it

\footnotetext{
2 Actually, for $\beta<1 / 2$ and $R_{\mathrm{c}}>R_{\mathrm{E}}$, between the additional radial critical curve and $R_{\mathrm{c}}$ there is also a tangential critical curve, but this feature is certainly a product of the small angle approximation in $\alpha_{\mathrm{r}}$, since it comes for very high refraction angles.
}

becomes effective at high densities. In the range of masses we are interested in, additional images would be formed only in optically thick clouds so that no signature of their existence would be visible on the light curves. On the other hand, decreasing the central mass and the mass of the cloud correspondingly, the role of refraction becomes prominent while the cloud becomes more and more transparent. Finally, going to the regime studied by Draine (1998) (no central mass and $M_{\mathrm{c}} \sim 10^{-3} M_{\odot}$ ), caustic-events start to be definitely observable. However, in PLAGs, the presence of a relevant central mass pushes the threshold given by Eq. (29) far beyond a high density regime, where the event just looks like a complete occultation (Fig. 2e).

Again, only in steep fronts is it possible to have radial critical curves as we saw in Sect. 3.4 for $\beta<1 / 2$.

\section{Physical nature of PLAGs}

In this section we examine the possible PLAG candidates, on the basis of the phenomenology discussed up to now. We have seen what the microlensing light curves should look like and we have established an optimal range for the parameters.

As regards cold PLAGs in the halo, i.e. MACHOs associated with gas clouds, they would be ideal candidates, since their size could be about of the same order of the Einstein radius. The existence of such objects in the halo would have very interesting consequences for our knowledge about the gas distribution in the halo. It could be argued that a large fraction of cold baryonic mass in the halo can be found as gas surrounding MACHOs, since the small masses of these objects would favour the stability of the clouds. If this is the case, the density of PLAGs may be comparable to ordinary MACHOs, and several PLAG events per year can be expected by observing towards the Magellanic Clouds.

Besides cold dark PLAGs in the halo, which surely represent the most interesting possibility, in this Section we will examine known types of nebulae to look for additional PLAG candidates.

Circumstellar disks are commonly found at IR wavelengths around young stars and around low mass and intermediate stars that are in their evolutionary stage from red giants to white dwarfs.

Proto-stars could be good candidates. In fact, they are isolated interstellar clouds undergoing gravitational collapse. The clouds are actually flattened to disks with circular or elliptical projected shape according to the tilt angle with respect to the line of sight. Proto-stars can be found in star-forming regions, usually located in the disk and in the spiral arms of the Milky Way. Of course, to observe light curves as those shown in Fig. 2, we are mainly interested in Proto-stars with $1<M / M_{\mathrm{c}}<10$ and $R_{\mathrm{c}} \sim$ $1-10^{2} \mathrm{AU}$.

In the last decade, a good number of Proto-stars have been detected in our Galaxy (see for instance 
Beckwith et al. 1990; Hillenbrand et al. 1992; McCaugherean \& O’Dell 1996; Schneider et al. 1999; Padgett et al. 1999; Tuthill et al. 2001) and, very recently, the HST found also a very compact star-forming region in LMC (Heydary-Malayeri et al. 2001). Twin protoplanetary disks, each of them with $R_{\mathrm{c}} \approx 20 \mathrm{AU}$, have also been observed (Giovanardi et al. 2000), suggesting the possibility of binary PLAG events.

Other good candidates could be the proto-planetary nebulae $(\mathrm{PPNe})$. They represent a transitory stage, between the asymptotic giant branch (AGB) and planetary nebulae phases, in the evolution of intermediate and low-mass stars. This stage is characterized by an expanding circumstellar envelope of gas surrounding a central star $(\mathrm{CPPN})$. In our phenomenological description, we can consider the PPNe as static systems, because the expansion velocity of the circumstellar envelope is $v_{\mathrm{e}} \approx 25 \mathrm{~km} \mathrm{~s}^{-1}$ (Osterbrock 1989). The typical mass of the $\mathrm{CPPN}$ is $M \approx 0.6 M_{\odot}$ and the mass of the circumstellar envelope is of the same order. Since they are expanding systems, it is certainly possible to observe PPNe with $R_{\mathrm{c}} \sim R_{\mathrm{E}}$. PPNe were only discovered and studied in the last twelve years, mostly thanks to IRAS and HST (Ueta et al. 2000). For a good overview about these objects, see Kwok (2000).

As well as in our galaxy, where we know roughly $10^{3}$ planetary nebulae $(\mathrm{PNe})$, intracluster $\mathrm{PNe}(\mathrm{IPNe})$ have also been detected thanks to the surveys towards the Virgo cluster (Mendez et al. 1997), M87 and its surrounding halo (Ciardulo et al. 1998; Feldemeier et al. 1998).

Besides Proto-stars and PPNe, we can consider other kinds of astrophysical objects that are associated with gas clouds. For instance, recently the VLT discovered a small cone-shaped nebula near the the old neutron star RX J1856.5-3754. This nebula extends for roughly 60 AU (van Kerkwijk 2001), and the neutron star is located very close to the top of the cone. Similar bow wave nebula have been found around other fast-moving supernova remnant, like IC 443 (Olbert et al. 2001), radio pulsars, like PSR B170644 (PSR J1709-4428) and PSR B1643-43 (PSR J16464346) (E. Giacani et al. 2001), nova system, like BZ Cam (Prinja et al. 2000), and could be good PLAG candidates.

All these objects are located mainly in the bulge, in the disk and in spiral arms of galaxies. So, good targets for the search of this kind of PLAGs are the Galactic bulge and rich star fields of the Milky Way spiral arms. However, the monitoring of other galaxies, like LMC or M 31, should give prominence to self-lensing PLAG events. It must be said that Protostars, PPNe and other exotic objects are not very common in the galaxy, so that their total optical depth in observations towards the bulge barely reaches $10^{-11}$ which is 5 orders of magnitude lower than disk star optical depth.

In a cosmological context, following the idea to find extra-galactic MACHOs by monitoring quasars behind the Virgo cluster of galaxies (Tadros et al. 1998), or by monitoring M 87 with the pixel lensing technique (Gould 1995), the search of intracluster PLAG events could give interesting results. However, considering the actual estimates of the abundance of intracluster nebulae (Ciardullo et al. 1998), the optical depth towards M 87 is around $10^{-12}$, which is still very low.

\section{Summary}

We studied gravitational microlensing due to point-like sources surrounded by diffuse gas clouds. Provided the size of the gas cloud is about of the order of the corresponding Einstein radius, we find that the presence of the gas cloud, via absorption or refraction effects, can substantially modify the classical Paczyński light curve and induce a strong chromaticity. The observation of these effects might not be easy, since they require a precise photometry and a continuos monitoring, as done for instance by the Planet and GMAN collaborations to find planets. Otherwise, if one looks for long duration events, the observations should last several years, in order to identify longer events.

We have hypothesized that cold dark PLAGs may be present in galactic halos and we have mainly focused on the determination of the characteristics they should have in order to be observable. If the existence of PLAGs in the halo is proved, then the recent estimates of baryonic dark matter should be revised to take into account the presence of gas clouds in the halo. Disk PLAG events, caused by known nebulae, or events caused by intracluster nebulae, should be very rare, if the estimates of their abundances are correct.

Due to the chromaticity, the present used selection criteria for microlensing reject PLAG events. It would thus be interesting to check whether already in the existing data there are PLAG microlensing events. We believe that a search for PLAG type events is necessary to complete the picture about baryonic dark matter in the halo.

Acknowledgements. V.B. and G.S. were supported by fund ex 60\% DPR 382/80 and F.S.E. of European Community. Ph.J. and L.M. acknowledge support from the Swiss National Science Foundation.

\section{References}

Alcock, C., Allsman, R. A., Alves, D. R., et al. 2000a, ApJ, 542,281

Alcock, C., Allsman, R. A., Alves, D. R., et al. 2000b, ApJ, 541,734

Beckwith, S. V. W., Sargent, A. I., Chini, R. S., \& Güsten, R. 1990, AJ, 99, 924

Cazes, J. E., \& Tohline, J. E. 2000, ApJ, 53, 1051

Chandrasekhar, S. 1960, Radiative Transfer (Dover Publications)

Ciardullo, R., Jacoby, G. H., Feldmeier, J. J., \& Bartlett, R. E. 1998, ApJ, 492, 62

De Paolis, F., Ingrosso, G., Jetzer, Ph., \& Roncadelli, M. 1995a, Phys. Rev. Lett., 74, 14

De Paolis, F., Ingrosso, G., Jetzer, Ph., \& Roncadelli, M. 1995b, A\&A, 295, 567 
De Paolis, F., Ingrosso, G., Jetzer, Ph., \& Roncadelli, M. 1996, Ap\&SS, 235, 329

De Paolis, F., Ingrosso, G., Jetzer, P., \& Roncadelli, M. 1998a ApJ, 500, 59

De Paolis, F., Ingrosso, G., Jetzer, P., \& Roncadelli, M. 1998b MNRAS, 294, 283

Derue, F., Afonso, C., Alard, C., et al. 2001, A\&A, 373, 123

Draine, B. T. 1998, ApJ, 509, L41

Feldemeier, J. J., Ciardulo, R., \& Jacoby, G. H. 1998, ApJ, 503,109

Gerhard, O., \& Silk, J. 1996, ApJ, 472, 34

Giacani, E. B., Frail, D. A., Goss, W. M., \& Vieytes, M. 2001, ApJ, 121, 3133

Giovanardi, C., Rodriguez, L. F., Lizanos, S., \& Canto, J. 2000, ApJ, 538, 728G

Gould, A. 1995, ApJ, 455, 44

Heydari-Malayeri, M., Rosa, M. R., Zinnecker, H., et al. 2001, A\&A, 344, 848

Hillenbrand, L. A., Strom, S. E., Vrba, F. J., \& Keene, J. 1992, ApJ, 397, 613

Kwok, S. 2000, The Origin and Evolution of Planetary Nebulae (Cambridge astrophysics series: 33)

Lasserre, T., Afonso, C., Albert, J. N., et al. 2000, A\&A, 355, L39

Mendez, R. H., Guerrero, M. A., Freeman, K. C., et al. 1997, ApJ, 491, L23
McCaughrean, M. J., \& O'Dell, C. R. 1996, AJ, 1111977

O'Dell, C. R., \& Beckwith, S. V. W. 1997, Science, 2761355

Padgett, D. L., Brandner, W., Stapelfeldt, K. R., et al. 1999, AJ, 117,1490

Pfenninger, D., Combes, F., \& Martinet, L. 1994, A\&A, 285, 79

Schneider, G., Smith, B. A., Becklin, E. E., et al. 1999, ApJ, 513, L127

Olbert, C. M., Clearfield, C. R., Williams, N. E., et al. 2001, ApJ, 554, L205

Osterbrock, D. E. 1989, Astrophysics of Gaseous Nebulae and Active Galactic Nuclei (University Science Books)

Prinja, R. K., Ringwald, F. A., Richard, A., \& Knigge, C. 2000, MNRAS, 312, 316

Rafikov, R. R., \& Draine, B. T. 2001, ApJ, 547, 207

Schneider, P., Ehlers, J., \& Falco, E. E. 1992, Gravitational Lenses (Springer Verlag)

Subramanian, K., \& Cowling, S. A. 1986, MNRAS, 219, 333

Tadros, H., Warren, S., \& Hewett, P. 1998, New Astron. Rev., 42, 115

Tuthill, P. G., Monnier, J. D., \& Danchi, W. C. 2001, Nature, 409, 1012

Ueta, T., Meixner, M., \& Bobrowsky, M. 2000, ApJ, 528, 861 van Kerkwijk, M. H. 2001, private communication

Woźniak, P. R., Udalski, A., Szymański, M., et al. 2001, Acta Astron. submitted [astro-ph/0106474] 